nach der Ruptur Ulzerationen ausbilden. Die Dauer der Erkrankung kann sechs bis acht Wochen betragen. Eine spezifische Therapie steht nicht zur Verfügung.

Kürzlich wurde der Fall einer Kuhpocken-Infektion eines zehnjährigen Mädchens aus Frankreich publiziert, bei dem ein ausgeprägter herpesähnlicher Befall der Vulva sowie orale Läsionen bestanden. Als Ansteckungsquelle wurden die eigenen Haustiere (Katze und Hund) angenommen, die jedoch klinisch unauffällig waren. Das Virus dürfte am ehesten per Autoinokulation in den Genitalbereich sowie in den Mund übertragen worden sein.

Klinisch wurde zunächst der fälschliche Verdacht auf eine Infektion mit Herpes simplex gestellt und eine spezifische antivirale Therapie eingeleitet. Erst durch die eher zufällig durchgeführte
Viruskultur ließ sich das Kuhpockenvirus anzüchten. Auch in der nach zwei Wochen durchgeführten Biopsie des genitalen Ulkus zeigte sich ein pockentypisches histologisches Bild.

Kommentar: Diese Fallbeschreibung erscheint auf den ersten Blick exotisch. Wegen der Seltenheit dürften allerdings viele Fälle - wie hier gezeigt - als „besonderer" Verlauf einer Herpes-genitalisoder Herpes-simplex-Infektion fehldiagnostiziert worden sein. In der Anamnese von lokalisierten Exanthemen, Vesikeln oder Ulzerationen sollte daher stets auch nach Tierkontakt (Katzen, Nagetiere) gefragt werden.

Prof. Dr. Tino F. Schwarz

Dina J et al. Genital ulcerations due to cowpox: a misleading diagnosis of herpes.

J Clin Virol 2011; 50: 345-7

\title{
Aggressive Tränen
}

Einer 89-jährigen Frau wurden Chloramphenicol-Augentropfen verordnet. Im Verlauf der folgenden zwei Behandlungswochen kam es zu einer allergischen Konjunktivitis und zu einem periorbitalen Erythem mit Schuppung.

$\mathrm{D}$ s Aussehen der Haut war charakteristisch für eine schwere Kontaktdermatitis und erstreckte sich auf einen Gesichtsbereich, über den die Tränen über die Wangen flossen. Somit musste man eine Auslösung durch die chloramphenicolhaltige Tränenflüssigkeit annehmen.

Eine lokale allergische Reaktion gegen Chloramphenicol kommt bei etwa einer von 200 behandelnden Personen vor. Zwar zeigen mehrere Fallberichte, dass Chloramphenicol selbst zu allergischen

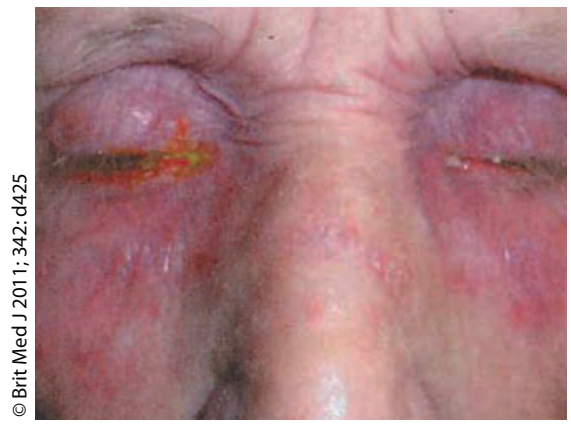

Reaktionen führen kann, häufiger sind jedoch Konservierungsmittel und Trägersubstanzen als Auslöser verantwortlich, im vorliegenden Fall wahrscheinlich Phenylquecksilbernitrat. Eine Hypersensitivitätsreaktion tritt in der Regel nur nach längerer Anwendung von chloramphenicolhaltigen Augentropfen auf. Die Hautveränderungen bilden sich meistens zurück, sobald die Therapie beendet wird.

Fazit: Das Verteilungsmuster von Hautkrankheiten ist ein wichtiges diagnostisches Hilfsmittel und erfordert manchmal das scharfe Denken von Sherlock Holmes - unter Beachtung aller Details.

Prof. Dr. Hermann S FüeßI

Chu C, Price N

Brit Med J 2011; 342: d425

Bei dieser Patienten haben chloramphenicolhaltige Tränen deutliche Spuren hinterlassen. 